\title{
Alimentação de Triportheus angulatus (Spix \& Agassiz, 1829) no lago Camaleão, Manaus, AM, Brasil.
}

\author{
Kedma Cristine YAMAMOTO ${ }^{1}$, Maria Gercilia Mota SOARES ${ }^{2}$, Carlos Edwar de Carvalho FREITAS ${ }^{3}$
}

\begin{abstract}
RESUMO
As inundações periódicas na Amazônia Central causam profundas modificações no meio ambiente. A alternância dos períodos de cheias e secas tem influência nos fatores bióticos e abióticos do meio aquático. Na enchente e na cheia é alta a oferta de alimentos, ampliando o espectro alimentar que é restrito na seca. As variáveis limnológicas também sofrem modificações. As concentrações de oxigênio têm variações sazonais e diárias, às vezes com períodos de hipoxia. Apesar dessas alterações, os lagos de várzea são habitados por muitas espécies de peixes e estão entre os ambientes de maior abundância e riqueza de peixes na Amazônia. A distribuição temporal e a alimentação de Triportheus angulatus (Spix \& Agassiz, 1829) foi estudada em um lago de várzea da Amazônia Central para entender a influência das modificações hídricas sobre o tamanho dos indivíduos, a composição da dieta e a ingestão dos alimentos mesmo em condições de baixas concentrações de oxigênio. Na enchente predominam os indivíduos menores, enquanto que na cheia, vazante e seca os maiores. Houve mudança sazonal na composição da dieta que foi relacionada com a disponibilidade de alimentos no ambiente: na enchente os peixes ingerem principalmente insetos e zooplâncton; na cheia e vazante frutos e sementes, e na seca insetos. O consumo de alimento foi alto na enchente e cheia, decrescendo na vazante e atingindo as menores quantidades na seca. A atividade alimentar, no período estudado, não foi influenciada pelas baixas concentrações de oxigênio existentes no lago.
\end{abstract}

\section{PALAVRAS-CHAVE}

Amazônia, áreas alagáveis, alimentação, Triportheus angulatus.

\section{Feeding of Triportheus angulatus (Spix \& Agassiz, 1829) in the Camaleão lake, Manaus, Amazonas state, Brazil.}

\begin{abstract}
Periodical flooding cause strong modifications in Central Amazonian floodplain. Periodical rising and decreasing of the water level influences abiotic and biotic factors of the aquatic environments. During the flooding season, there is a high feed availability, in contrast with dry season in which feed availability is at the lowest level. Limnological parameters also change seasonally. Oxygen concentrations vary both daily and seasonally, sometimes even showing hypoxic conditions. Despite these alterations, floodplain lakes are inhabited by several fish species, having the most diverse and abundant fish fauna among the Amazonian environments. This study focused on Triportheus angulatus feeding in temporal distribution in order to understand the influences of bydrological changes on individual size, diet composition and feeding even in low oxygen concentrations. In rising waters season, small individuals are predominant while the decreasing and the dry seasons there are mostly larger ones. There is a seasonal change on diet composition and this is related to feed type that is more available: during the rising, fishes eat mainly insects and zooplankton; in the flood season and drying season, seeds and fruits are predominant, and at the dry season they eat mainly insects. Feed ingestion was higher in the rising waters and mostly in the flood, and it was lower in the drying season. Feeding activity was not related with oxygen concentrations.
\end{abstract}

\section{KEY WORDS \\ Amazon, floodplain areas, feeding, Triportheus angulatus.}

\footnotetext{
${ }^{1}$ Centro Universitário Luterano de Manaus - CEULM/ ULBRA, Departamento de Ciências Biológicas. Av. Solimões, $\mathrm{n}^{\circ} 2$, conj. Atílio Andreazza, Bairro Japiim II, 69077-730, Manaus - Amazonas, Brasil, fax 236-7817, yamamoto@inpa.gov.br

${ }^{2}$ Instituto Nacional de Pesquisas da Amazônia - INPA. Caixa Postal, 478, 69011-090, Manaus - Amazonas, Brasil, 643-3269, gesoares@inpa.gov.br

${ }^{3}$ Departamento de Ciências Pesqueiras, Faculdade de Ciências Agrárias, Universidade Federal do Amazonas - UFAM, Av. Gen. Otávio Ramos Jordão, 300 - CEP 69077-000, Manaus - Amazonas, Brasil, cefreitas@ufam.edu.br
} 


\section{ACTA AMAZONICA}

ALIMENTAÇÃO DE Triportheus angulatus (SPIX \& AGASSIZ, 1829) NO LAGO CAMALEÃO, MANAUS, AM, BRASIL

\section{INTRODUÇÃO}

$\mathrm{Na}$ Amazônia as áreas inundáveis dos grandes rios de água branca, como o Amazonas e seus tributários Purus e Juruá, atingem cerca de $200.000 \mathrm{~km}^{2}$ e são denominadas regionalmente como várzeas (Junk, 1993). Estas áreas são muito produtivas e ao serem inundadas aumentam a diversidade de habitats que são colonizados por uma rica ictiofauna.

Na Amazônia Central a flutuação do nível d'água, média anual de $10 \mathrm{~m}$, promove a alternância das fases terrestre e aquática resultando em modificações drásticas nas condições ambientais, exigindo adaptações específicas da ictiofauna (Junk et al., 1983). Na época de enchente e cheia, o aumento da área alagada favorece a exploração de vários habitats incrementando o espectro alimentar dos peixes (Santos, 1981; Almeida, 1984; Soares et al, 1986). Entretanto, as condições favoráveis são limitadas pelas flutuações sazonais e nictemerais nas concentrações de oxigênio com períodos de hipóxia ou anóxia (Schmidt, 1973; Junk et al., 1983; SouzaPereira, 2000). Na vazante e seca, a retração das águas limita o espaço, obrigando os peixes a migrarem da várzea para outros locais como o canal principal dos rios ou para os lagos permanentes (Lowe-McConnel, 1964; Goulding, 1980).

Dentre os peixes que habitam as áreas alagadas, destacamse as espécies do gênero Triportbeus, sardinhas, por sua ampla distribuição e adaptabilidade a vários biótopos. As sardinhas estão entre os sete peixes mais comercializados nos mercados de Manaus (Batista, 1998; Soares \& Junk, 2000). Foram realizados diversos trabalhos sobre os peixes dos rios Machado, Madeira e Negro elucidando os hábitos alimentares de espécies locais e evidenciando suas interações com a área alagável (Goulding, 1980; Soares et al., 1986; Goulding et al., 1988); sobre a composição da dieta relacionada com a morfologia (Almeida, 1984); e ainda descrições taxonômicas (Gery, 1977) e estudos sobre os aspectos reprodutivos (Fernandes, 1988; Braga, 1990).

Na Amazônia Central, o lago Camaleão é povoado por uma ictiofauna rica (Bayley 1982; Junk et al., 1983; SouzaPereira, 2000) destacando-se Triportheus angulatus pela abundância e ampla distribuição no lago sendo, inclusive, capturada em locais com baixas concentrações de oxigênio. Com base nestas informações, supõe-se que T. angulatus tenha desenvolvido mecanismos adaptativos para habitar os lagos. Neste trabalho é investigado como varia a alimentação de T. angulatus frente às modificações hídricas que influenciam na disponibilidade de alimentos em um lago de várzea, mesmo sob condições de baixas concentrações de oxigênio.

\section{ÁREA DE ESTUDO}

O lago Camaleão localiza-se na ilha da Marchantaria, no Município de Iranduba, Amazonas, Brasil nas coordenadas 03ำ'S e 59⒌'W. A ilha está situada cerca de $15 \mathrm{~km}$ acima da confluência dos rios Solimões e Negro (Fig.1 A). A flutuação média anual do nível da água varia em torno de
$10 \mathrm{~m}$. Normalmente, a enchente no rio começa em novembro e atinge a cota máxima em julho; a vazante iniciase em agosto quando a água flui do lago para o rio, sendo outubro o mês mais seco (Fig.1B).

\section{MATERIAL E MÉTODOS}

Os exemplares de T. angulatus foram coletados em quatro períodos do ciclo hidrológico: enchente (março a abril), cheia (maio a julho), vazante (agosto a setembro) e seca (setembro a janeiro) (Fig. 1B) de abril de 1998 a abril de 2000. Os peixes foram capturados com malhadeiras de diferentes tamanhos variando entre 30 e $70 \mathrm{~mm}$ entre nós opostos, que foram estendidas das margens para o centro do lago de forma a abranger diferentes habitats. As
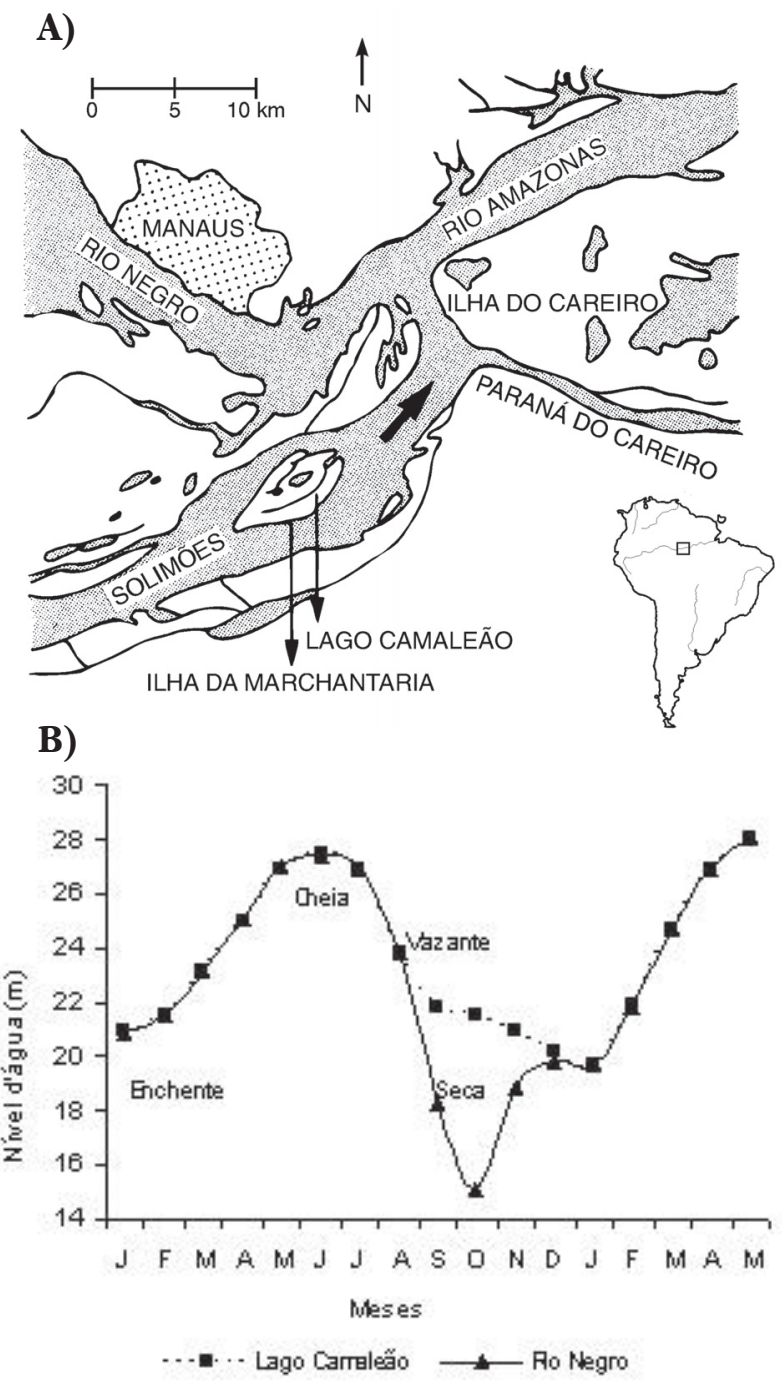

Figura 1 - Localização do lago Camaleão na ilha da Marchantaria (A) (Furch\& Junk, 1993) e variação mensal do nível da água do rio Negro e do lago Camaleão em 1998 (B). 


\section{ACTA AMAZONICA}

malhadeiras ficaram expostas durante 24 horas, com despescas a cada 6 horas. Os exemplares capturados foram acondicionados em gelo, transportados para o laboratório de Biologia e Ecologia de Peixes de Áreas Alagadas do INPA, onde foram registrados os dados biométricos, peso total (g) e comprimento padrão $(\mathrm{cm})$. Concomitante às coletas dos peixes foram medidas as concentrações de oxigênio na superfície, meio e fundo do lago por meio de um oxímetro, WTW, modelo OXI 96, com precisão de $0,1 \mathrm{mg} /$, equipado com sensor em cabo de $20 \mathrm{~m}$.

Os estômagos retirados dos tratos digestivos foram examinados sob microscópio estereoscópio e os itens alimentares identificados até o mais preciso nível taxonômico possível. Nestas análises foram utilizados os métodos de freqüência de ocorrência e de volume relativo (Goulding 1980; Hyslop, 1980). Os resultados foram combinados no índice alimentar (IAi) (Kawakami \& Vazzoler, 1980) para evidenciar os itens alimentares mais importantes da dieta. Os valores do índice alimentar foram transformados em percentagem.

$$
I A i=\frac{F i \cdot V i}{\sum_{i=1}^{n}(F i \cdot V i)}
$$

onde:

$\mathrm{i}=$ item alimentar;

$\mathrm{Fi}=$ freqüência de ocorrência (\%) do iésimo item alimentar;

$\mathrm{Vi}=$ volume (\%) do iésimo item alimentar.

Para determinar os padrões alimentares de T. angulatus, foi efetuada uma Análise de Correspondência (Braak, 1995) usando como objetos os períodos do ciclo hidrológico (enchente, cheia, vazante e seca) e como descritores os principais itens alimentares.

A determinação do período de atividade alimentar foi efetuada através da identificação do grau de repleção estomacal dos exemplares por meio de uma avaliação visual do grau de enchimento dos estômagos. Os estômagos foram abertos em uma placa de Petri e o grau de preenchimento avaliado conforme a seguinte escala: 0\% (vazio), 25\%, 50\%, 75\% e 100\%.

A estrutura de comprimento da população foi obtida a partir da distribuição da freqüência relativa das classes de comprimento padrão $(4 \mathrm{~cm})$ dos peixes amostrados em cada período hidrológico. Os exemplares foram agrupados em 6 classes de comprimento: 0-4, 4-8, 8-12, 12-16, 16-20 e >20 cm.

\section{RESULTADOS}

De abril de 1998 a abril de 2000 foram capturados 1196 exemplares de T. angulatus com comprimento padrão variando de 3 a $25 \mathrm{~cm}$ no lago Camaleão. A maioria dos exemplares incluiu-se nas classes de $8-12 \mathrm{~cm}(40 \%)$ e de $12-16 \mathrm{~cm}$ (39\%), seguidas das classes de $4-8 \mathrm{~cm}$ (16\%) e de $16-20 \mathrm{~cm} \mathrm{(5 \% ).} \mathrm{Peixes} \mathrm{com} \mathrm{comprimento} \mathrm{entre} \mathrm{8-12} \mathrm{cm}$ foram predominantes na enchente $(51 \%)$ e entre $12-16 \mathrm{~cm}$ na cheia (74\%), vazante (58\%) e seca (60\%). Os exemplares menores, $4-8 \mathrm{~cm}$, foram freqüentes na enchente (20\%) e os maiores $16-20 \mathrm{~cm}$, na cheia (20\%) e vazante (22\%). Somente $2 \%$ dos peixes capturados na vazante apresentaram comprimento $>20 \mathrm{~cm}$ (Fig. 2).

A análise do conteúdo estomacal de 363 exemplares indicou uma dieta constituída por zooplâncton (copépodos, cladóceras e rotíferos), insetos (insetos imaturos, larvas e ninfas, insetos adultos, himenópteros, pedaços de coleópteros, dípteros, hemípteros e pedaços de insetos), frutos/sementes (embaúba, Cecropia latiloba; tarumã, Vitex cimosa e catoré, Crateva benthami), restos vegetais (pedaços de folhas, galhos e raízes) e algas (unicelulares, coloniais e filamentosas). Os valores do Índice Alimentar indicam os insetos (52,7\%), frutos/sementes (24,2\%) e zooplâncton $(19,6 \%)$ como os alimentos de maior importância na dieta de T. angulatus no lago.

A dieta de T. angulatus variou sazonalmente sendo constituída principalmente de insetos e zooplâncton na enchente, dos frutos/sementes na cheia e vazante e dos insetos na seca (Fig. 3). Em cada período hidrológico a composição da dieta de T. angulatus foi similar entre os exemplares das
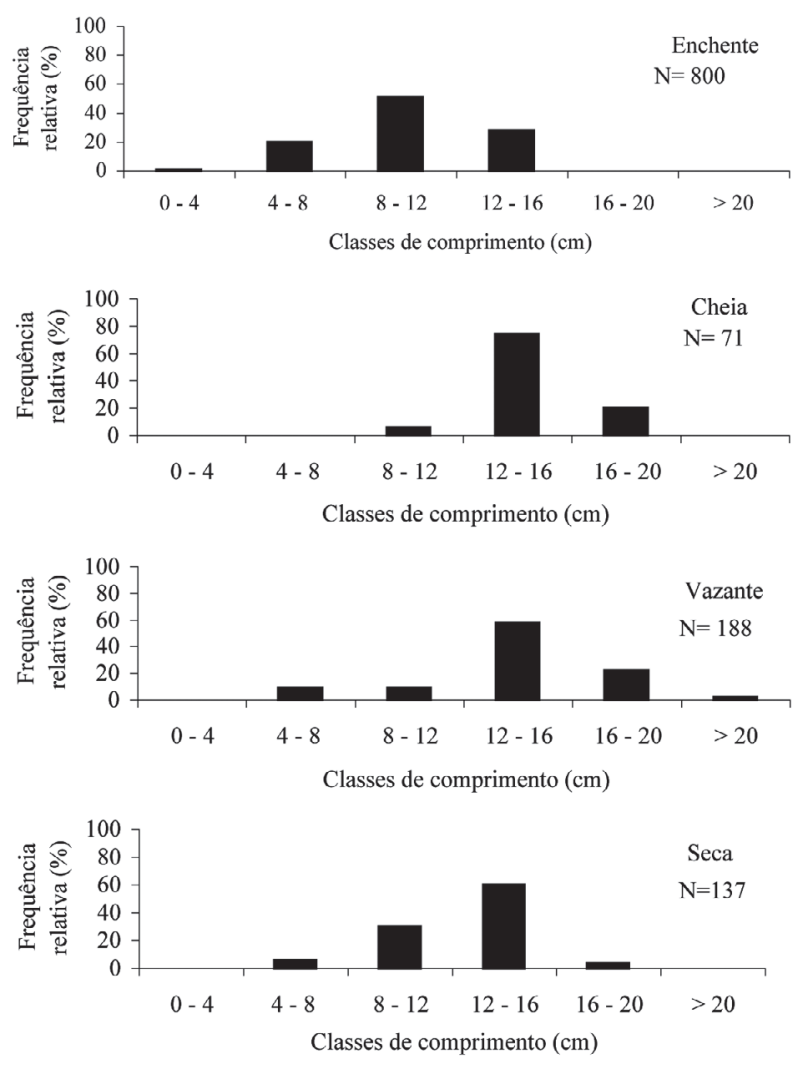

Figura 2 - Variação sazonal das classes de comprimento $(\mathrm{cm})$ de T. angulatus no lago Camaleão. 
diferentes classes de comprimento. Uma exceção no período $\mathrm{da}$ enchente foram os exemplares da classe de $4-8 \mathrm{~cm}$ que ingeriram apenas insetos, enquanto que os peixes das classes 8-12 e 12-16 cm, ingeriram insetos e zooplâncton.

A análise de correspondência mostrou que no período de enchente ocorreu uma sobreposição de itens alimentares, indicando que neste período a disponibilidade de alimento é maior (zooplâncton, algas, restos vegetais, frutos/sementes e insetos) em relação às outras épocas do ano, concordando com os dados de composição da dieta. Frutos/sementes é o alimento com maior disponibilidade na enchente, cheia e vazante, posicionando-se próximo ao eixo de ambos os períodos (Fig. 4). Já no eixo da seca é clara a redução na disponibilidade de alimentos, sendo insetos e material vegetal os mais consumidos (Fig. 4). Assim sendo, o padrão alimentar de T. angulatus em relação aos descritores (itens alimentares) e aos objetos (enchente, cheia, vazante e seca) é sazonal por explorar determinados alimentos em diferentes épocas do ano.

Observou-se que entre os 1196 exemplares de T. angulatus examinados mais da metade (52\%) não continham alimento nos estômagos. Nos períodos de enchente e cheia foi maior a percentagem de estômagos com alimento, decrescendo na vazante e alcançando os menores valores na seca (Tab. 1).
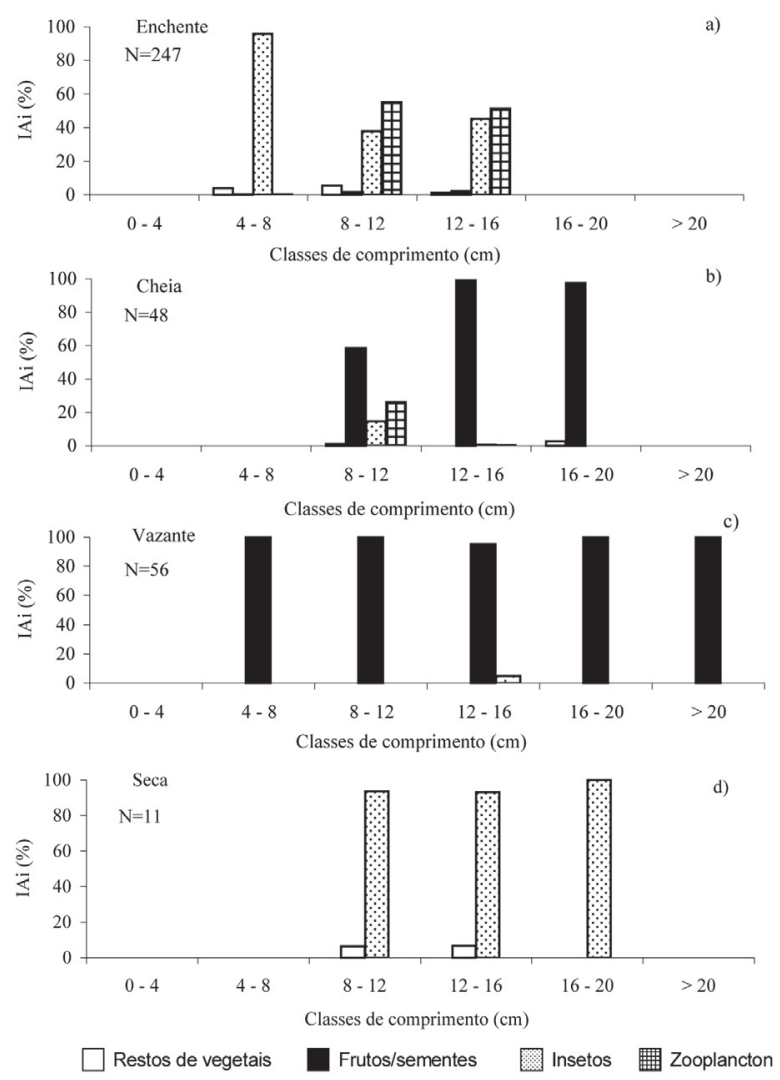

Figura 3 - Variação sazonal do Índice Alimentar (IAi) dos principais itens alimentares identificados nos estômagos de T.angulatus
De maneira geral, a atividade alimentar foi similar entre as diferentes classes de comprimento em cada período, exibindo pouca variação em relação à quantidade de alimento ingerido (Fig. 5). $\mathrm{Na}$ enchente os peixes das classes de comprimento 4-8, 8-12 e 12-16 cm tinham cerca de 52\% dos estômagos com alimentos enquanto que, nas de 0-4 e 16-20 prevaleceram estômagos vazios. Entretanto, o número de exemplares nestas duas últimas classes é muito baixo (Fig. 5a). Na cheia, mais de $70 \%$ dos peixes em todas as classes, apresentaram estômagos com alimentos (Fig. 5b). Na vazante a percentagem de exemplares com alimento nos estômagos das classes de 4-8, 8-12 e 12-16 cm (37 a 42\%) foi menor do que nas classes $16-20$ e $>20 \mathrm{~cm}$ (71 a 75\%) (Fig. 5c). Na seca 67 a $100 \%$ dos exemplares em todas as classes de comprimentos estavam com estômagos vazios (Fig. 5d).

A concentração de oxigênio na superfície da água do lago foi em geral baixa, especialmente nos períodos de enchente $(0,4 \mathrm{mg} / \mathrm{l})$ e cheia $(1,2 \mathrm{mg} / \mathrm{l})$, contudo aumentou na vazante $(1,5 \mathrm{mg} / \mathrm{l})$ e alcançou os maiores valores na seca $(3,4 \mathrm{mg} / \mathrm{l})$. Em profundidades maiores que $1 \mathrm{~m}$ a água esteve praticamente anóxica durante os períodos de enchente, cheia e vazante (Tab. 2).

\section{DISCUSSÃO}

No lago Camaleão alevinos e juvenis $(0-12 \mathrm{~cm})$ de $T$. angulatus são capturados em abundância na época de enchente enquanto na vazante e seca há incremento no número de exemplares de maior tamanho $(12-20 \mathrm{~cm})$. Este fato está relacionado com os movimentos reprodutivos que ocorrem no início da enchente em direção à calha central dos rios (Goulding, 1980; Fernandes, 1988), que favorecem a dispersão de larvas e alevinos carreados pela correnteza para as áreas de inundação após o período de desova (Araújo-Lima, 1984; Petry, 1989; Lima, 2002). A estratégia reprodutiva dos Characiformes migradores de desovar a

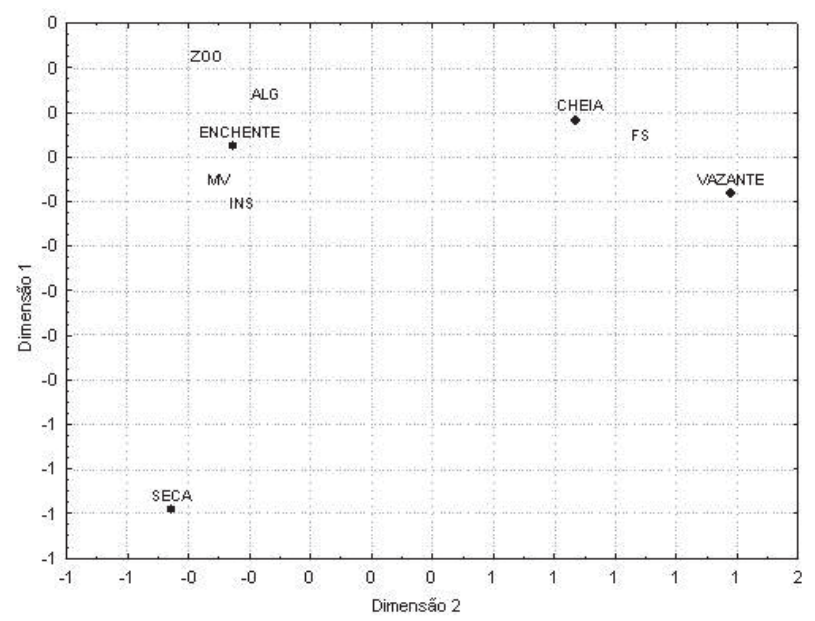

Figura 4 - Análise de correspondência dos principais itens alimentares de T. angulatus em um ciclo hidrológico. 


\section{ACTA AMAZONICA}

montante dos rios de águas brancas, provavelmente está direcionada a posicionar as larvas para crescerem nas áreas de inundação onde ocorre o aumento da oferta de alimentos (Araújo-Lima, 1984). Embora não haja certeza de que os ovos e larvas de peixes tenham sido levados para a ilha da Marchantaria, o tamanho da população indica que ocorre um grande movimento de alevinos de sardinhas no lago Camaleão durante a enchente do rio Solimões.

Tabela 1 - Número (N) e freqüência de ocorrência (\%) de estômagos vazios e com alimentos de T. angulatus.

\begin{tabular}{lccccc}
\hline \hline Períodos & \multicolumn{3}{c}{ Vazios } & \multicolumn{3}{c}{ Com alimentos } & \\
\cline { 2 - 5 } & $\mathbf{N}$ & $\%$ & $\mathrm{~N}$ & $\%$ & Total \\
\hline Enchente & 390 & 49 & 410 & 51 & 800 \\
Cheia & 20 & 28 & 51 & 72 & 71 \\
Vazante & 103 & 55 & 85 & 45 & 188 \\
Seca & 113 & 82 & 24 & 18 & 137 \\
\hline \hline
\end{tabular}
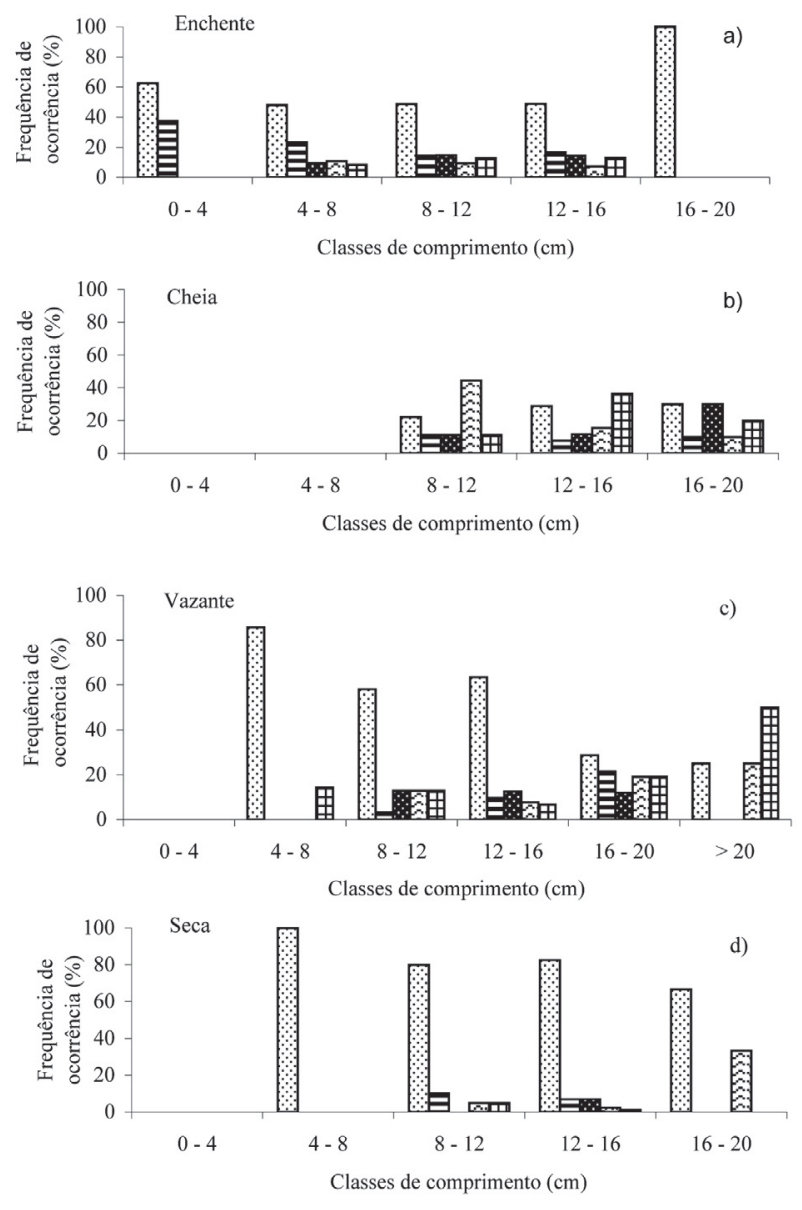

응 $0 \%$ 日 $0-25 \%$ - $250 \%$ 日 $50-75 \%$ 日 $75-100 \%$

Figura 5 - Distribuição da freqüência relativa dos graus de repleção dos estômagos de T. angulatus. Em cada período é dado o número de exemplares correspondente a cada classe de comprimento.
T. angulatus permanece no lago Camaleão durante o período alagado, porém, com a retração das águas os indivíduos quase adultos começam a sair do lago antes do isolamento total do lago em relação ao rio Solimões. No entanto, alguns exemplares podem ficar presos no lago durante o período de isolamento. No lago do Rei vários Characiformes, inclusive as sardinhas, realizam migrações laterais para fora da várzea $\mathrm{e}$ iniciam no rio Solimões uma migração longitudinal rio acima (Fernandes, 1988). Por causa destes movimentos a pesca profissional de sardinhas apresenta características sazonais, com picos de abundância na vazante e início da seca (agosto, setembro e outubro) (Batista, 1998; Soares \& Junk, 2000).

A dieta de T. angulatus no lago Camaleão é constituída por vários itens alimentares, sendo os frutos/sementes, insetos, zooplâncton e restos vegetais os de maior freqüência $\mathrm{e}$ abundância. A composição tem variação sazonal sendo elevado consumo de insetos e zooplâncton na enchente. Os insetos são disponibilizados durante a alagação, especialmente as formigas por causa da grande quantidade de colônias espalhadas nos galhos, folhas e troncos caídos das árvores, tornando-se assim presas fáceis para peixes de hábito pelágico. O zooplâncton aumenta sua abundância e riqueza na época da enchente decrescendo na seca (Koo, 2000). Na cheia e vazante os peixes ingerem muitos frutos/sementes de Cecropia latiloba (embaúba), Vitex cimosa (tarumã) e Crateva benthami (catoré). As árvores disponibilizam esse alimento por vários meses, pois o período de frutificação se estende de maio (final da enchente) (Kubitzki \& Ziburski, 1994) a setembro (vazante) (Worbes, 1997). Na seca, a retração das águas resulta em uma drástica diminuição da área alagada, forçando os peixes a migrarem dos outros habitats em direção à água aberta do lago. Esta situação influencia a composição da dieta dos peixes, por causa da redução na oferta de alimentos em comparação com outras épocas do ano. Assim, T. angulatus ingere itens de origem vegetal e insetos, que são os alimentos mais disponíveis nesse período de escassez.

T. angulatus é altamente adaptado (morfologia) a ingerir frutos/sementes e invertebrados que caem na superfície da água. Observa-se que ele explora com eficiência a floresta alagada durante o período de alagação, concordando com os resultados obtidos em outros estudos realizados no lago Janauacá (Almeida, 1984), no rio Uatumã (Leite, 1987) e no rio Machado (Goulding, 1980).

Estudos sobre alimentação de espécies de peixes onívoras amazônicas mostram uma variação sazonal dos itens alimentares (Goulding, 1980; Almeida, 1984). A composição da dieta de muitas espécies de peixes parece estar relacionada com a dinâmica do pulso de inundação das áreas alagáveis. Mylossoma duriventre, na Amazônia Central, apresenta variação sazonal da dieta, ingerindo frutos e sementes, material vegetal e zooplâncton na cheia e principalmente insetos na seca (Yamamoto, 2001).

A variação ontogênica da dieta foi baixa, uma vez que esta foi similar entre as classes de comprimento em cada período do ciclo hidrológico. No entanto, na enchente os indivíduos menores $(4-8 \mathrm{~cm})$ ingeriram principalmente 
Tabela 2 - Variação sazonal da concentração de oxigênio na coluna d'água.

\begin{tabular}{lccc}
\hline \hline Periodo & \multicolumn{3}{c}{ Profundidade } \\
\cline { 2 - 4 } & Superficie & Meio & Fundo \\
\hline Enchente & 0,4 & 0,2 & 0 \\
Cheia & 1,2 & 0 & 0 \\
Vazante & 1,5 & 0,1 & 0,1 \\
Seca & 3,4 & 2,9 & 2,8 \\
\hline \hline
\end{tabular}

insetos, enquanto que os maiores $(8-12$ e $12-16 \mathrm{~cm})$ ingeriram insetos e zooplâncton. Isto provavelmente está relacionado com a distribuição dos exemplares no lago: os menores concentram-se nas margens e os maiores realizam movimentos entre as margens e água aberta.

A atividade alimentar está diretamente relacionada com a variação sazonal do ciclo hidrológico, que altera a disponibilidade dos itens alimentares. Na enchente e especialmente durante a cheia (as águas altas) ocorre maior disponibilidade de alimentos, junto com a dispersão dos peixes nos diversos habitats, intensificando a atividade alimentar. $\mathrm{O}$ contrário ocorre na vazante e seca, quando a acentuada redução da atividade alimentar pode ser explicada pela escassez de alimentos. A menor quantidade de alimentos pode ser compensada pela qualidade, por exemplo, os itens alimentares de origem animal muitas vezes têm maior valor nutritivo do que os de origem vegetal. A fim de compensar os períodos de maior escassez de alimentos, os peixes armazenam gordura nas cavidades abdominais durante os períodos de abundância (Junk, 1985).

As menores quantidades de alimento encontradas nos estômagos durante a seca estão em concordância com os resultados obtidos em outros estudos (Lowe-McConnell, 1964; Goulding, 1980; Goulding \& Carvalho, 1982) que relacionam o período de seca como a época de restrição alimentar para muitas espécies de peixes.

Basile-Martins (1978) constatou que a maior incidência de estômagos vazios ocorre em indivíduos mais velhos. Neste caso a ingestão de alimentos estaria relacionada com o estádio de desenvolvimento sexual, os peixes jovens alimentam-se com maior freqüência do que peixes adultos. Para T. angulatus não foi possível constatar diferenças na atividade alimentar entre as classes de comprimento, provavelmente por que a maioria dos peixes capturados no lago ainda é juvenil. Somente na vazante os peixes acima de $16 \mathrm{~cm}$, ingeriram maiores quantidades de alimentos.

A alimentação é freqüentemente afetada pela redução de oxigênio, visto que a procura, digestão e assimilação são os maiores componentes do balanço energético em muitos peixes (Brett \& Groves, 1979). Porém, as condições de baixas concentrações de oxigênio não afetaram a ingestão de alimentos em T. angulatus. Na enchente e na cheia quando as concentrações de oxigênio são reduzidas, os exemplares foram capturados em locais anóxicos, tanto na floresta alagada quanto na água aberta, porém, com estômagos contendo alimentos: frutos/sementes, insetos e restos vegetais. De fato T. angulatus é uma espécie pelágica que tem mecanismos adaptativos como a alta tolerância às baixas concentrações de oxigênio. Experimentos em aquários relatam que em concentrações abaixo de $0,4 \mathrm{mg}$ $\mathrm{O}_{2} / 1$. angulatus inicia a respiração na superfície da água auxiliada por barbelas e pelo crescimento dermal da mandíbula (Braum \& Junk, 1982; Saint-Paul \& Soares, 1987; Winemiller, 1989). Esta adaptação não só permite a sobrevivência dos peixes nos períodos de hipóxia e anóxia, mas também a manutenção da atividade básica que lhes fornecem energia e alimentação. Ao ser mantido o nível de ingestão é garantido o desenvolvimento normal do seu ciclo vital.

O lago Camaleão pode ser considerado um ambiente de alimentação e crescimento para T. angulatus. No lago há disponibilidade de alimento ao longo de todo o ciclo hidrológico, e as condições de hipóxia e anóxia, características de lagos de várzea da Amazônia Central, não são fatores limitantes para T. angulatus.

\section{AGRADECIMENTOS}

Ao Instituto Nacional de Pesquisas da Amazônia (INPA), Max-Planck-Institute für Limology, Working Group for Tropical Ecology, Plön, Alemanha e o projeto PP-G7/PPD (No 1161/99) pelo suporte financeiro.

\section{BIBLIOGRAFIA CITADA}

Araújo-Lima, C. A. R. M. 1984. Distribuição espacial e temporal de larvas de Characiformes em um setor do rio Amazonas, próximo a Manaus, Am. Dissertação de Mestrado. Instituto Nacional de Pesquisas da Amazônia/Fundação Universidade do Amazonas, Manaus, Amazonas. 82p.

Almeida, R. G. 1984. Biologia alimentar de três espécies de Triportheus (Pisces: Characoidei: Characidae) do lago Castanho, Amazonas. Acta Amazonica, 14(1): 48-76.

Batista, V. S. 1998. Distribuição, dinâmica da frota e dos recursos pesqueiros da Amazônia Central. Tese de Doutorado. Instituto Nacional de Pesquisas da Amazônia/Fundação Universidade do Amazonas, Manaus, Amazonas. 291p.

Bayley,P. B. 1982. Central Amazon fish populations: biomass, production and some dynamic characteristics. - $\mathrm{Ph}$. D. These, Dalhousie University, Canada. 308p.

Basile-Martins, M. A. 1978. Comportamento e alimentação de Pimelodus maculatus LÁCEDE, 1803 (Osteichthyes, Siluriformes, Pimelodidae). Tese de Doutorado, USP, São Paulo. 143p.

Braga, F.M.S. 1990. Aspectos da reprodução e alimentação de peixes comuns em um trecho do rio Tocantins entre Imperatriz e Estreito, Estados do Maranhão e Tocantins, Brasil. Revta. Brasil. Biol., 50 (3): 547-558.

Braak, C. J. F. T. 1995. Ordination. In: Jongmam, R. H. G; Braak, C. J. F. T.; Torgeren, O. F. R. V. (eds.) Data Analysis in Community and Landscape Ecology, Cambride University Press, USA: 91-173.

Braum, E.; Junk, W.J. 1982. Morphological adaptation of two Amazonian characoids (Pisces) for surviving in oxygen efficient waters. Int. Revue ges. Hydrobiol., 67:869-886. 


\section{ACTA AMAZONICA}

Brett, J. R.; Groves, T. D. D. 1979. Physiological energetics. In: Hoar, W. S.; Randall, D. J.; Brett, J. R. (ed.) Fish Physiology. vol. 08, Academic Press, New York: 279-352.

Fernandes, C.C. 1988: Estudos sobre migrações laterais de peixes no sistema do lago do Rei (Ilba do Careiro) Am- Br. Dissertação de Mestrado, Instituto Nacional de Pesquisas da Amazônia/Fundação Universidade do Amazonas, Manaus, Amazonas. 170p.

Furch, K.; Junk, W. J. 1993. Seasonal nutrient dynamics in an Amazonian floodplain lake. Arch. Hydrobiol., 128(3): 277-285.

Gery, J. 1977. Characoids of the wold. TFH Publications, Neptune City, 672p.

Goulding, M. 1980. The fishes and the forest: Explorations in Amazonian Natural History. University of California Press: Berkeley, CA, USA. 280p.

Goulding, M.; Carvalho, M.L. 1982. Life history and management of the Tambaqui, (Colossoma macropomum, Characidae): An important Amazonian food fish. Revta. Bras. Zool., 1: 107-133.

Goulding, M.; Carvalho M. L.; Ferreira E. G. 1988. Rio Negro: rich life in poor water: Amazonian diversity and foodplain ecology as seen through fish communities. SPB Academic Publishing The Hague. 200p.

Hyslop, E.J. 1980. Stomach contents analysis review of methods and their applications. J. Fish Biol., 17: 411-429.

Junk, W.J. 1985. Temporary fat storage, and adaptation of some fish species to the waterlevel fluctuations and related environmental changes of the Amazon river. Amazoniana, IX: 315-351.

Junk, W.J. 1993. Wetlands of Tropical South America. In: Whigham, D., Hejny, S.; Dykyjova, D. (eds.) Wetlands of the world. Dr. W. Junk Publ., Dordrecht, Boston: 679-739.

Junk, W. J.; Soares, M.G.M; Carvalho, F. M. 1983. Distribution of fish species in a lake of the Amazon river floodplain near Manaus (Lago Camaleão) with special reference to extreme oxygen conditions. Amazoniana, 7(4): 397 - 431.

Kawakami, E.; Vazzoler, G. 1980. Métodos gráficos e estimativa de índice alimentar aplicado no estudo de alimentação de peixes. Bol. Inst. Oceanogr., 29(2): 205-207.

Koo, F.W.C. 2000. Interações peixes/zooplâncton no lago Camaleão, um lago de várzea da Amazônia Central, Brasil. Dissertação de Mestrado, Instituto Nacional de Pesquisas da Amazônia/Fundação Universidade do Amazonas, Manaus, Amazonas. 91p.

Kubitzki, K.; Ziburski, A. 1994. Seed dispersal in flood plain forests of Amazonia. Biotropica, 26 (1): 30-43.

Leite, R.G. 1987. Alimentação e hábitos alimentares dos peixes do rio Uatumã. Dissertação de Mestrado, Instituto Nacional de Pesquisas da Amazônia/Fundação Universidade do Amazonas, Manaus, Amazonas. 81p.

Lima, A.C. 2002. A desova dos characiformes do rio Solimões/ Amazonas. Dissertação de Mestrado, Instituto Nacional de Pesquisas da Amazônia/Fundação Universidade do Amazonas, Manaus, Amazonas. 38p.
Lowe-McConnell, R.H. 1964. The fishes of the Rupunini Savana district of British Guiana, South America. I. Ecological of fish species and effects of the seasonal cycle on the fish. J. Linn. Soc. (Zool), 45(304): 103-144.

Petry, P. 1989. Deriva de Ictioplâncton no Paraná do Rei, várzea do Careiro, Amazônia Central, Brasil. Dissertação de Mestrado, Instituto Nacional de Pesquisas da Amazônia / Fundação Universidade do Amazonas, Manaus, Amazonas. 67p.

Santos, G. M. 1981. Estudos de alimentação e hábitos alimentares de Schizodon fasciatus Agassiz, 1829, Rhytiodus microlepis Kner, 1859 e Rhytiodus argentofuscus Kner, 1859, do lago Janauacá - Am. Acta Amazonica, 11: 267-283.

Saint-Paul, U.; Soares, M. G. M. 1987. Diurnal distribution and behavioral responses of fishes to extreme hypoxia in an Amazon floodplain lake. Env. Biol. Fish., 20: 91-104.

Soares, M.G.M., Almeida, R.G.; Junk, W.J. 1986. The tropic status of the fish fauna in lago Camaleão e macrophyte dominated Floodplain Lake in the middle Amazon. Amazoniana, 9(4): 511-526.

Soares, M.G.M.; Junk, W.J. 2000. Commercial Fishery and Fish Culture of the State of Amazonas: Status and Perspectives. In: Junk, W.J., Ohly, J.J., Piedade, M.T.F.; Soares, M.G.M. (eds.). The Central Amazon Floodplain: actual use and options for a sustainable management. Backhuys Publishers b.V., Leiden: 433-461.

Souza-Pereira, A. 2000. Variáveis limnológicas e sua relação com as espécies de peixes onívoras e herbivoras do lago Camaleão, Amazonas, Brasil. Monografia, Fundação Universidade do Amazonas, Manaus, Amazonas. 31 p.

Schmidt, G. W. 1973. Primary production of phytoplankton in the three types of Amazonian waters. II. The limnology of a tropical floodplain lake in Central Amazonian (Lago do Castanho). Amazoniana, 4 (2):139-203.

Winemiller,K.O. 1989. Development of dermal lip protuberances for aquatic surface respiration in South American characid fishes. Copeia 1989(2):382-390.

Worbes, M. 1997: The forest ecosystem of the floodplains. Junk, W. J. (ed.) The Central Amazon floodplain. Ecology of a pulsing system. Ecological Studies, Vol. 126, Springer, Berlin: 223-226.

Yamamoto, K. C.; Oliveira, A. C. B.; Soares, M. G. M. 2001. Distribuição e Alimentação de Mylossoma duriventre no Lago Camaleão, Amazônia Central, Manaus, AM. Anais do XXIII Congresso de Zoologia, Universidade Federal de Cuiabá - MT: 410-411.

\footnotetext{
RECEBIDO EM: 01/10/2003

ACEITO EM: 13/10/2004
} 
